\title{
RENDIMIENTO DE LÍNEAS DE MAÍZ EXÓTICO IRRADIADO CON RAYOS GAMMA Y DE HÍBRIDOS DE CRUZA SIMPLE
}

\author{
YIELD OF EXOTIC MAIZE LINES IRRADIATED WITH GAMMA \\ RAYS AND OF SINGLE CROSS HYBRIDS
}

\author{
Moisés Buenrostro-Robles ${ }^{1}$, Ricardo Lobato-Ortiz ${ }^{1}$, \\ J. Jesús García-Zavala' y Carlos Sánchez-Abarca ${ }^{2}$
}

\begin{abstract}
'Postgrado en Recursos Genéticos y Productividad-Genética, Campus Montecillo, Colegio de Postgraduados. km 36.5 Carr. México-Texcoco. 56230, Montecillo, Estado de México, México. ${ }^{2}$ Departamento de Fitotecnia, Universidad Autónoma Chapingo. km 38.5 Carr. México-Texcoco. 56230. Chapingo, Estado de México.
\end{abstract}

*Autor para correspondencia (rlobato@colpos.mx)

\section{RESUMEN}

La generación de híbridos de maíz (Zea mays L.) de alto rendimiento para Valles Altos es necesaria ante la creciente demanda de grano. En el mejoramiento genético esta actividad tiene un atractivo más cuando las líneas progenitoras provienen de germoplasma exótico. Con el objetivo de determinar el rendimiento potencial per se de líneas de maíz exótico irradiado con $\mathrm{Co}^{60}$ y de híbridos simples que se generaron con ellas, se evaluaron 53 líneas progenitoras derivadas de 10 líneas de alto potencial de rendimiento altamente homocigóticas, seis cruzas simples y dos híbridos comerciales como testigos. La evaluación se realizó en 2015 en tres ambientes: Montecillo, Chapingo y Tecámac, Estado de México, bajo un diseño experimental de bloques completos al azar con tres repeticiones. La unidad experimental constó de parcelas de dos surcos de $6 \mathrm{~m}$ de largo, con $80 \mathrm{~cm}$ entre surcos y $40 \mathrm{~cm}$ entre plantas. Se registraron los días a floración masculina (DFM); altura de planta y mazorca (AP, AM), en $\mathrm{cm}$; rendimiento de grano ( $t \mathrm{ha}^{-1}$ ) (RG); longitud y diámetro de mazorca (LMz, DMz) en $\mathrm{cm}$; número de hileras de granos por mazorca (NHM); número de granos por hilera (NGH). Hubo significancia entre ambientes, entre genotipos, y en la interacción genotipo $\times$ ambiente para todas las variables. El RG de las 10 mejores líneas superó $3.4 \mathrm{t} \mathrm{ha}^{-1}$, lo que muestra su potencial como progenitores de híbridos de cruza simple. Las cruzas $99 \times 107,107 \times 206$ y $106 \times 180$ fueron las más precoces con rendimientos de $8.432,8.432$ y $8.276 \mathrm{t} \mathrm{ha}^{-1}$, respectivamente, $y$ estadísticamente diferentes a los híbridos testigo HS-2 (9.421 t ha-1) y H-40 $\left(8.913 \mathrm{t} \mathrm{ha}^{-1}\right)$. Las líneas más productivas podrían representar una ventaja en la producción de semilla híbrida de menor costo de aquellas cruzas simples de alto potencial productivo.

Palabras clave: Zea mays, híbridos, germoplasma exótico, irradiación recurrente.

\section{SUMMARY}

The development of maize hybrids (Zea mays L.) with high yield for the Mexican highlands is necessary to face the increasing demand for grain. In genetic improvement, this activity has additional attractiveness when parental lines come from exotic germplasm. In order to determine the per se potential performance of exotic maize lines irradiated with $\mathrm{Co}^{60}$ and simple hybrids generated with them, 53 progenitor lines derived from 10 highly homozygous high yielding lines, along with six single crosses and two commercial hybrids as checks were evaluated. The evaluation was carried out in 2015 in three environments: Montecillo, Chapingo and Tecamac, State of Mexico, under a randomized complete blocks design with three replications. The experimental unit consisted of plots with two $6-\mathrm{m}$ long rows, $80 \mathrm{~cm}$ between rows and 40 $\mathrm{cm}$ between plants. The traits measured were days to male flowering (DFM) plant and ear height (AP, AM) in cm, grain yield $\left(\mathrm{t} \mathrm{ha}^{-1}\right)(\mathrm{RG})$; ear length and ear diameter ( $\mathrm{LMz}, \mathrm{DMz})$ in $\mathrm{cm}$; number of kernel rows per ear (NHM); number of grains per row (NGH). There was significance between environments, genotypes, and genotype $\times$ environment interaction for all the evaluated traits. $\mathrm{RG}$ of the 10 best lines exceeded $3.4 \mathrm{t} \mathrm{ha}^{-1}$, which shows their potential as parents of single cross hybrids. The crosses $99 \times 107,107 \times 206$ and $106 \times$ 180 were the most early-maturing with yields of $8.432,8.432$ and $8.276 \mathrm{tha}^{-1}$, respectively, and statistically different from the check hybrids HS-2 $(9.421 \mathrm{t}$ $\left.\mathrm{ha}^{-1}\right)$ and $\mathrm{H}-40\left(8.913 \mathrm{t} \mathrm{ha}^{-1}\right)$. The most productive lines could represent an advantage in the production of low cost hybrid seed of those single crosses with high productive potential.

Index words: Zea mays, hybrid, exotic germplasm, recurrent irradiation.

\section{INTRODUCCIÓN}

El maíz (Zea mays L.) se ha colocado como el cultivo más importante del mundo por el volumen de producción de 1037.7 millones de toneladas (FAOSTAT, 2015) y por ser la fuente de alimentación de millones de humanos, de animales, y de materias primas para la industria, por lo cual su mejoramiento genético es muy importante. Desafortunadamente, la preferencia de los fitomejoradores a determinados grupos de germoplasma élite ha conducido a un estancamiento en los avances y alcances de los programas existentes de mejoramiento genético del maíz (Hallauer, 1994). Así, frecuentemente los programas de mejoramiento genético para formar nuevas variedades mejoradas usan un espectro restringido de germoplasma localmente adaptado y disponible dentro de las regiones productoras de maíz, como son la templada, tropical, subtropical, y la de los valles altos (Goodman, 1992; Hallauer, 1992).

Los programas de mejoramiento genético dedicados a la formación de híbridos y variedades comerciales de maíz 
requieren generar nuevas líneas con alto potencial de rendimiento, buen comportamiento agronómico y buena aptitud combinatoria. Se ha encontrado que las líneas que reúnen estas características presentan resultados satisfactorios en combinaciones híbridas (Fan et al., 2003). Al respecto, Arellano et al. (2011) mencionan que es factible, en función del potencial genético del híbrido, la utilización de genotipos que mantengan un comportamiento estable en diferentes localidades y años, así como un rendimiento alto.

El elevado costo de la semilla de híbridos de cruza simple de líneas endogámicas de bajo rendimiento per se ha limitado su producción comercial en México (Luna et al., 2012), por lo que en su lugar se usan híbridos de cruza trilineal o doble. En el caso de Estados Unidos, se utilizan híbridos de cruza simple en la producción comercial de maíz porque disponen de líneas autofecundadas con alto rendimiento per se (Hallauer et al., 1988).

Debido a que en los Valles Altos de México se usan principalmente variedades de maíz de las razas Chalqueño y Cónico, para impulsar la diversidad genética del maíz en la región y aumentar su productividad, en el Colegio de Postgraduados se inició un programa de mejoramiento genético por selección recurrente con autofecundación e irradiación de germoplasma tropical exótico (CervantesSantana et al., 2002). La finalidad de tal proyecto fue la identificación de germoplasma exótico con nuevos alelos favorables ausentes en las poblaciones locales, para usarlo per se o para transferir sus alelos y aprovecharlos en líneas de maíz y en los híbridos generados. Por lo tanto, el objetivo del presente trabajo fue evaluar el rendimiento de líneas per se derivadas de germoplasma tropical exótico seleccionado e irradiado en valles altos y de algunos de sus híbridos de cruza simple y compararlo con el de híbridos comerciales para riego. Se planteó la hipótesis de que el comportamiento per se para rendimiento de grano de las líneas y de los híbridos será diferente entre ellos y a través de ambientes, lo que dará la oportunidad de seleccionar los mejores materiales para recomendar su uso en programas de mejoramiento genético del maíz en Valles Altos.

\section{MATERIALES Y MÉTODOS}

Los materiales genéticos consistieron de 53 líneas progenitoras derivadas de 10 líneas de maíz de alto rendimiento y altamente homocigóticas (Cuadro 1), seis cruzas simples y los híbridos comerciales HS-2 y H-40 usados como testigos. Las 10 líneas de alto rendimiento, de las que se derivaron las 53 líneas de este trabajo, fueron generadas a partir de poblaciones de maíces tropicales, originalmente tardías, adaptadas por selección a valles altos. Estas poblaciones se sembraron a densidades de 200,000 plantas ha-1 y se formaron previamente mediante cruzas intervarietales de compuestos de razas tropicales de maíz para obtener líneas altamente productivas, precoces (Pérez-López et al., 2014) y con un alto coeficiente de endogamia (0.996); esta metodología se repitió por ocho ciclos y además se aplicó irradiación gama recurrente de Cobalto 60 entre cada ciclo de selección, para generar mutaciones y seleccionar aquellas favorables en las plantas. Con el propósito de mostrar la base genética de las 53 líneas evaluadas, en el Cuadro 1 se presenta la genealogía de sus 10 líneas progenitoras.

\begin{tabular}{|c|c|}
\hline Línea & Genealogía \\
\hline 1 & [Reventador $\left(\right.$ Sin 60) × Olotón (Chis 284)] $\mathrm{F}_{18}$ \\
\hline 2 & [Chapalote $(\operatorname{Sin} 2) \times$ Tuxpeño $\left(\right.$ Oax 9)] $F_{25}$ \\
\hline 3 & $\mathrm{RTM}^{+}$- Comp. 1B (0.5-1.0)-5-2-3-16\# \\
\hline \multirow[t]{2}{*}{4} & $\mathrm{CRT}^{\bullet}$ 15R1M1-20R4M1-15R1M1-11R1M11 \\
\hline & 14R1M2-18R1M1-25R1M11-2744-61-24\# \\
\hline 5 & [Reventador $\left(\right.$ Sin 60) × Vandeño $\left(\right.$ Chis 80)] $F_{18}$ \\
\hline 6 & RTM - Comp. 1B (0.5-1.0)-1-1-3-17\# \\
\hline 7 & [Tuxpeño $\left(\right.$ Oax 9) × Olotillo (Chis 114] $\mathrm{F}_{25}$ \\
\hline 8 & [Blandito (Sin 61) $\times$ Pepitilla (Mor 102)] $\mathrm{F}_{25}$ \\
\hline 9 & RTM - Comp. 1B (0.5-1.0)-5-3-3-24\# \\
\hline 10 & RTM - Comp. 1 A (0.5-1.0)-7-2-3-19\# \\
\hline
\end{tabular}


La evaluación de los 61 materiales se realizó en el ciclo primavera-verano 2015 en tres ambientes: Montecillo y Chapingo, Estado de México, donde prevalece un clima tipo Cw (i) B/2 (a') templado con lluvias en verano; precipitación media anual de $655.6 \mathrm{~mm}$; temperatura media anual de $16.3^{\circ} \mathrm{C}$, y Tecámac, Estado de México con un clima del tipo Cw (i) B/2 (a') templado, semiseco con lluvias en verano; precipitación media anual de $636 \mathrm{~mm}$ y temperatura media anual de $16.4^{\circ} \mathrm{C}$. Las tres localidades se ubican a una altitud entre 2200 y 2300 m, propia de los Valles Altos de México.

El diseño experimental utilizado fue de bloques completos al azar con tres repeticiones; la parcela experimental constaba de dos surcos de $6 \mathrm{~m}$ de largo y $80 \mathrm{~cm}$ de ancho con 32 matas de dos plantas sembradas cada $40 \mathrm{~cm}$, equivalente a una densidad de población de 62,500 plantas ha $^{-1}$. La siembra se realizó de manera manual en MonteciIlo (29/abril/2015), Chapingo (20/mayo/2015) y Tecámac (27/mayo/2015). Ésta se realizó en suelo seco y se regó inmediatamente después de sembrar, después se aplicaron riegos de auxilio suficientes hasta el establecimiento de las Iluvias. La fertilización se hizo con 120 kg N y 80 $\mathrm{kg} \mathrm{P}_{2} \mathrm{O}_{5}$ ha- $^{-1}$, el $\mathrm{P}_{2} \mathrm{O}_{5}$ fue aplicado en su totalidad junto con la mitad del $\mathrm{N}$ en la siembra, y el resto del $\mathrm{N}$ se aplicó al realizar la primera de dos escardas (30 días después de la siembra, dds). Para el control de maleza se aplicó Gesaprim ${ }^{\circledR}$ Calibre 90 GDA, $\left(3 \mathrm{~L} \mathrm{ha}^{-1}\right)$ a los 15 dds.

Las variables evaluadas fueron: rendimiento de grano $(R G)$, el cual se estimó con el peso de las mazorcas y plantas cosechadas por unidad experimental, cuyo contenido de humedad fue ajustado a $12 \%$, multiplicado por el índice de desgrane y transformado a $\mathrm{kg} \mathrm{ha}^{-1}$; días a floración masculina (DFM); altura de planta (AP, cm) y altura de mazorca $(A M, c m)$. En una muestra de cinco mazorcas por unidad experimental se registraron las siguientes variables: longitud de mazorca ( $L M z, c m)$, diámetro de mazorca $(\mathrm{DMz}, \mathrm{cm})$ tomado en la parte media de la misma; número de hileras por mazorca (NHM), y número de granos por hilera (NGH).

Se efectuaron análisis de varianza por ambiente y uno combinado donde la fuente de variación Genotipos se desglosó en Líneas, Cruzas, Testigos, y Grupos de Materiales; también se hizo una prueba de comparación de medias (Tukey, 0.05) para cada una de las variables; ambos procedimientos se hicieron mediante el paquete SAS versión 9.3 (SAS Institute, 2002). El modelo estadístico para el análisis de varianza combinado fue:

$$
Y_{i j k l}=\mu+A_{k}+R_{l(k)}+G_{i}+A G_{i k}+£_{i j k l}
$$

donde, $Y_{i j k l}=$ valor del $j$-ésimo genotipo al ser evaluado en el k-ésimo ambiente en la l-ésima repetición; $\mu$ = media general; $A_{k}=$ efecto atribuido al $k$-ésimo ambiente; $R_{(k)}=$ efecto de repetición anidada en el k-ésimo ambiente; $G_{j}=$ efecto del genotipo; $A G_{i k}=$ efecto de interacción genotipo $\times$ ambiente; $y €_{i j k l}=$ efecto aleatorio atribuido al error experimental.

\section{RESULTADOS Y DISCUSIÓN}

El análisis de varianza combinado a través de ambientes (Cuadro 2) detectó diferencias altamente significativas ( $P$ $\leq 0.01$ ) entre ambientes, entre genotipos y en la partición entre líneas, entre cruzas, entre testigos, y entre grupos de materiales para todas las variables, excepto para días a floración masculina (DFM) y número de hileras por mazorca (NHM). La interacción genotipo × ambiente también resultó altamente significativa para todas las variables.

La significancia entre ambientes indica que las condiciones climáticas y agroecológicas variaron de una localidad a otra aun en el mismo año de cultivo, lo que se reflejó en la diferente expresión promedio de las variables en cada ambiente. En especial se presentaron granizadas en Montecillo que dañaron a las plantas durante su crecimiento y también ocurrieron ataques de pájaros que mermaron el rendimiento, aparte de las diferencias en precipitaciones y temperaturas entre las localidades. Tales condiciones meteorológicas afectaron la expresión de los genotipos para las variables evaluadas de una localidad a otra.

La significancia entre genotipos evidencia que los materiales experimentales tuvieron diferente expresión fenotípica, debido a las diferencias genéticas intrínsecas entre los genotipos, los cuales provienen de diferentes orígenes geográficos y raciales; por la misma razón, la partición de genotipos también resultó significativa para todas las variables en los grupos de materiales de interés de este trabajo.

Por otro lado, la interacción significativa genotipo $\times$ ambiente indica que los materiales interaccionaron con los ambientes debido a su respuesta diferencial a los efectos ambientales, esto como resultado de su diferente constitución genética y expresión fenotípica. Los efectos adversos del clima por daños de granizadas antes de la floración, los daños por pájaros y las diferencias en precipitación y suelo entre localidades fueron los que principalmente causaron la interacción genético-ambiental.

La comparación de medias por ambiente indica que los genotipos $106 \times 180,105 \times 194$ y $107 \times 206$ evaluados en Chapingo tuvieron el mayor rendimiento de grano $(P \leq$ $0.05)$, con $8.420 \mathrm{t} \mathrm{ha}^{-1}, 8.392 \mathrm{t} \mathrm{ha}^{-1}$ y $8.318 \mathrm{t} \mathrm{ha}^{-1}$, respectivamente; en Montecillo los rendimientos fueron de $9.705 \mathrm{t}$ 
Cuadro 2. Cuadrados medios del análisis de varianza para rendimiento de grano y sus componentes de 61 genotipos de maíz evaluados en tres ambientes. Ciclo agrícola primavera-verano, 2015.

\begin{tabular}{lrcccccccr}
\hline FV & GL & RG & DFM & AP & AM & LMz & DMz & NHM & NGH \\
\hline AMB & 2 & $10,885,537 * *$ & $621 * *$ & $97,923.10 * *$ & $45327 * *$ & $13.30 * *$ & $1.55 * *$ & $39.83 * *$ & $56.60 * *$ \\
REP(AMB) & 6 & $3080 \mathrm{~ns}$ & $1.17 \mathrm{~ns}$ & $4.33 \mathrm{~ns}$ & $4.30 \mathrm{~ns}$ & $0.002 \mathrm{~ns}$ & $0.007 \mathrm{~ns}$ & $0.10 *$ & $0.23 \mathrm{~ns}$ \\
GEN & 60 & $42,199,257 * *$ & $52.40 * *$ & $5737 * *$ & $2103.50 * *$ & $18.46 * *$ & $1.50 * *$ & $11.30 * *$ & $145.40 * *$ \\
LIN & 52 & $8,399,668 * *$ & $43.50 * *$ & $4392.50 * *$ & $1584 * *$ & $11.30 * *$ & $1.00 * *$ & $6.31 * *$ & $92.80 * *$ \\
CRUZ & 5 & $5,390,557 * *$ & $5 * *$ & $1141 * *$ & $1253.10 * *$ & $2.70 * *$ & $0.10 * *$ & $1.00 * *$ & $19.50 * *$ \\
TEST & 1 & $1,160,470.30 *$ & $0.22 \mathrm{~ns}$ & $365 *$ & $430.22 *$ & $0.27 *$ & $0.22 *$ & $0.01 *$ & $44.50 * *$ \\
GRUP & 2 & $1,033,529,728 * *$ & $429 * *$ & $54,868.10 * *$ & $18,574.40 * *$ & $252.12 * *$ & $20.01 * *$ & $171.34 * *$ & $1880 * *$ \\
AMB $\times$ GEN & 120 & $1,596,594 * *$ & $24 * *$ & $624.02 * *$ & $397.10 * *$ & $3.45 * *$ & $0.16 * *$ & $1.60 * *$ & $18.40 * *$ \\
Error & 360 & 3343 & 0.67 & 9.62 & 7.54 & 0.003 & 0.005 & 0.040 & 0.14 \\
\hline
\end{tabular}

**: altamente significativo con $\mathrm{P} \leq 0.01$, * Significativo con $\mathrm{P} \leq 0.05$, ns: no significativo; FV: fuente de variación; GL: grados de libertad; RG: rendimiento de grano ( ha $\left.^{-1}\right)$; DFM = días a floración masculina; AP. altura de planta; AM: altura de mazorca; LMz: longitud de mazorca; DMz: diámetro de mazorca; NHM: número de hileras por mazorca; NGH: número de granos por hilera.

ha $^{-1}, 8.966$ t ha-1 $^{-1} 8.658$ t ha-1 $^{-1}$, para los genotipos $99 \times 107$, $106 \times 180$ y $107 \times 206$, respectivamente, y en Tecámac de $8.281 \mathrm{t} \mathrm{ha}^{-1}, 7.512 \mathrm{t} \mathrm{ha}^{-1}$ y $7.495 \mathrm{t} \mathrm{ha}^{-1}$, para los genotipos $107 \times 206,109 \times 205$ y $105 \times 194$; lo cual señala que dentro de los tres genotipos con los mejores rendimientos para cada ambiente destaca el genotipo $107 \times 206$ con un promedio superior a las $8.2 \mathrm{t} \mathrm{ha}^{-1}$ para los tres ambientes, lo que representa una buena alternativa de uso para siembras comerciales de valles altos, por rendimiento y precocidad (73 días a floración, Cuadro 5). Los componentes del rendimiento resultaron estadísticamente diferentes entre los ambientes, en los que destacan como los mejores RG y LMz, en Chapingo (Cuadro 3).

Los resultados anteriores se debieron a que en Chapingo las condiciones ambientales fueron las más favorables para el desarrollo del cultivo, lo cual favoreció que los materiales genéticos $106 \times 180,105 \times 194$ y $107 \times 206$ presentaran, en promedio, la mejor expresión en las variables RG, LMz y DMz; con un rendimiento de $8.420 \mathrm{t} \mathrm{ha}^{-1}, 8.392 \mathrm{t} \mathrm{ha}^{-1}$ y 8.318 t ha $^{-1}$, respectivamente (datos no presentados). En contraste, en Montecillo el daño de pájaros y la caída de granizo poco antes de la floración afectaron el rendimiento de grano, por lo que este resultó menor que en Chapingo; sin embargo, por ser Montecillo el ambiente donde se formaron los genotipos, en esta localidad se obtuvieron las mayores expresiones $(P \leq 0.05)$ para todas las demás variables. Al respecto, De Brito et al. (2011) indican que daños severos del área foliar en plantas de maíz comprometen su productividad.

En Tecámac, por otro lado, al ser un ambiente más restrictivo, se tuvo un menor desarrollo del cultivo, sobre todo en etapas críticas como la floración y llenado de grano donde hubo restricción de humedad, lo que influyó en el rendimiento y sus componentes de los genotipos evaluados. Estas diferencias ambientales contribuyen a causar diferencias en los días a floración y rendimiento de grano, como lo reportaron Virgen-Vargas et al. (2016) al evaluar cruzas simples de maíz para valles altos en tres años. Efectos similares de los factores ambientales en maíz fueron observados por Boomsma et al. (2009), Ramírez et al. (2010) y Gómez-Espejo et al. (2015) en el sentido de que un ambiente desfavorable contribuye a las diferencias entre ambientes en los días para el inicio de la floración y en el rendimiento de grano.

En los tres ambientes se detectó una separación de los grupos de poblaciones (líneas, cruzas y testigos) (Cuadro 4); el grupo 3, formado por los híbridos comerciales testigo, presentó en los tres ambientes el rendimiento de grano más alto, seguido por el grupo 2, formado por las mejores 6 cruzas simples; la diferencia en rendimiento por hectárea fue $1.067,2$ y $0.551 \mathrm{t} \mathrm{ha}^{-1}$ para Montecillo, Chapingo y Tecámac, respectivamente; el rendimiento de grano se mantuvo estable en los tres ambientes. Para el caso de las cruzas simples, éstas tuvieron rendimientos de 7.5 a $8.0 \mathrm{t} \mathrm{ha}^{-1}$; Hallauer et al. (2010) mencionan que las cruzas simples presentan mayor interacción con el ambiente al ser poblaciones de condición más homogénea en comparación con los híbridos trilineales y dobles. Gómez-Espejo et al. (2015) al evaluar poblaciones de maíz de amplia base genética adaptadas a valles altos, encontraron que estas poblaciones interaccionaron poco con los ambientes debido a la presencia de una mayor frecuencia de genes favorables para adaptación; sin embargo, el rendimiento resulta ser la variable más afectada por los factores adversos del clima. Por lo anterior, es necesario conocer dicho efecto del ambiente en la productividad tanto de las líneas progenitoras como de las cruzas simples de híbridos de 
Cuadro 3. Comparación de medias entre tres ambientes para rendimiento de grano y sus componentes de 61 genotipos de maíz. Ciclo agrícola primavera-verano 2015.

\begin{tabular}{lllllllll}
\hline Ambiente & RG & DFM & AP & AM & LMz & DMz & NHM & NGH \\
\hline Montecillo & $3259 \mathrm{~b}$ & $77.3 \mathrm{a}$ & $205.2 \mathrm{a}$ & $107.5 \mathrm{a}$ & $13.28 \mathrm{~b}$ & $4.25 \mathrm{a}$ & $13.07 \mathrm{a}$ & $22.41 \mathrm{a}$ \\
Chapingo & $3305 \mathrm{a}$ & $74.0 \mathrm{c}$ & $182.4 \mathrm{~b}$ & $91.0 \mathrm{~b}$ & $13.50 \mathrm{a}$ & $4.25 \mathrm{a}$ & $12.88 \mathrm{~b}$ & $21.54 \mathrm{~b}$ \\
Tecámac & $2862 \mathrm{c}$ & $76.1 \mathrm{~b}$ & $159.0 \mathrm{c}$ & $76.0 \mathrm{c}$ & $12.96 \mathrm{c}$ & $4.09 \mathrm{~b}$ & $12.19 \mathrm{c}$ & $21.37 \mathrm{c}$ \\
DSH $_{0.05}$ & 14.20 & 0.20 & 0.80 & 0.70 & 0.01 & 0.01 & 0.04 & 0.09 \\
\hline
\end{tabular}

Medias con letras iguales dentro de columnas no son estadísticamente diferentes (Tukey, 0.05). RG: rendimiento de grano por hectárea (t ha-1); DFM: días a floración masculina; AP. altura de planta; AM: altura de mazorca; LMz: longitud de mazorca (cm); DMz: diámetro de mazorca (cm); NHM: número de hileras por mazorca; NGH: número de granos por hilera; DSH: diferencia significativa honesta (0.05).

Cuadro 4. Promedios del rendimiento de grano y sus componentes por grupo de materiales genéticos de maíz dentro de cada ambiente. Ciclo agrícola primavera-verano 2015.

\begin{tabular}{lccccccccc}
\hline Ambiente & Gpo & RG & DFM & AP & AM & LMz & DMz & NHM & NGH \\
\hline Montecillo & 1 & $2533.50 \mathrm{c}$ & $78 \mathrm{~b}$ & $198.30 \mathrm{~b}$ & $104.60 \mathrm{~b}$ & $12.90 \mathrm{~b}$ & $4.10 \mathrm{c}$ & $12.90 \mathrm{~b}$ & $21.40 \mathrm{c}$ \\
Montecillo & 2 & $7796.60 \mathrm{~b}$ & $72 \mathrm{c}$ & $249.50 \mathrm{a}$ & $123.30 \mathrm{a}$ & $15.60 \mathrm{a}$ & $4.77 \mathrm{~b}$ & $13.20 \mathrm{~b}$ & $27.60 \mathrm{~b}$ \\
Montecillo & 3 & $8863.60 \mathrm{a}$ & $81.50 \mathrm{a}$ & $253.70 \mathrm{a}$ & $135 \mathrm{a}$ & $16.10 \mathrm{a}$ & $5.40 \mathrm{a}$ & $16.70 \mathrm{a}$ & $32.25 \mathrm{a}$ \\
DSH $_{0.05}$ & & 1042.00 & 3.40 & 24.80 & 14.80 & 1.20 & 0.28 & 1.00 & 3.20 \\
Chapingo & 1 & $2491.30 \mathrm{c}$ & $74 \mathrm{~b}$ & $177.40 \mathrm{~b}$ & $87.30 \mathrm{~b}$ & $13.10 \mathrm{~b}$ & $4.20 \mathrm{c}$ & $12.70 \mathrm{~b}$ & $20.60 \mathrm{c}$ \\
Chapingo & 2 & $8039.10 \mathrm{~b}$ & $70.70 \mathrm{c}$ & $217 \mathrm{a}$ & $117.20 \mathrm{a}$ & $15.87 \mathrm{a}$ & $4.65 \mathrm{~b}$ & $12.70 \mathrm{~b}$ & $26.85 \mathrm{~b}$ \\
Chapingo & 3 & $10677.30 \mathrm{a}$ & $80.30 \mathrm{a}$ & $212 \mathrm{a}$ & $105.30 \mathrm{a}$ & $15.84 \mathrm{a}$ & $5.30 \mathrm{a}$ & $17.30 \mathrm{a}$ & $31.30 \mathrm{a}$ \\
DSH $_{0.05}$ & & 1066.00 & 2.80 & 20.00 & 14.80 & 1.30 & 0.40 & 0.90 & 3.40 \\
Tecámac & 1 & $2154.20 \mathrm{~b}$ & $76 \mathrm{~b}$ & $154.30 \mathrm{~b}$ & $73 \mathrm{~b}$ & $12.60 \mathrm{~b}$ & $4.00 \mathrm{c}$ & $12.00 \mathrm{~b}$ & $20.40 \mathrm{c}$ \\
Tecámac $^{2}$ & 2 & $7410.10 \mathrm{a}$ & $76.30 \mathrm{~b}$ & $184.20 \mathrm{a}$ & $98 \mathrm{a}$ & $15.30 \mathrm{a}$ & $4.66 \mathrm{~b}$ & $12.40 \mathrm{~b}$ & $25.50 \mathrm{~b}$ \\
Tecámac $^{2}$ & 3 & $7962 \mathrm{a}$ & $80.20 \mathrm{a}$ & $204.30 \mathrm{a}$ & $95.70 \mathrm{a}$ & $16.20 \mathrm{a}$ & $5.30 \mathrm{a}$ & $17.00 \mathrm{a}$ & $33.60 \mathrm{a}$ \\
DSH $_{0.05}$ & & 913.10 & 2.20 & 21.30 & 14.80 & 1.30 & 0.30 & 0.80 & 3.50 \\
\hline
\end{tabular}

Medias con letras iguales dentro de columnas para cada ambiente no son estadísticamente diferentes (Tukey, 0.05). Gpo 1: líneas; Gpo 2: cruzas; Gpo 3: testigos; RG: rendimiento de grano por hectárea (t ha-1); DFM: días a floración masculina; AP. altura de planta (cm); AM: altura de mazorca $(\mathrm{cm})$; LMz: longitud de mazorca (cm); DMz: diámetro de mazorca $(\mathrm{cm})$; NHM: número de hileras por mazorca; NGH: número de granos por hilera; DSH: diferencia significativa honesta (0.05).

maíz para determinar el sitio, fecha y la manera de establecer un lote de producción de semilla (Virgen-Vargas et al., 2014). Ávila et al. (2009) observaron un efecto ambiental similar al incrementar la cruza simple hembra del híbrido H-52 en el Valle de Texcoco, cuya producción de grano fue de 9 a 10 t ha- $^{-1}$

En el grupo 1, formado por líneas endogámicas se obtuvo el rendimiento de grano más bajo (Cuadro 4). Ésto debido a que han experimentado entre cinco y siete autofecundaciones, por lo que alelos con efectos deletéreos podrían estar en condición homocigótica y causar depresión endogámica, lo que afecta la expresión de algunas características que influyen en el rendimiento de grano.

El largo y diámetro de mazorca de los grupos 2 y 3 en los tres ambientes fueron similares, caso contrario con el número de hileras por mazorca y número de granos por hilera, donde el grupo 3 tuvo una marcada diferencia superior con respecto al grupo 2 y éste sobre el grupo 1 . Esto explica el rendimiento de grano más alto del grupo 3, ya que de acuerdo con Andrade et al. (1996) el número de granos por mazorca es un componente importante del rendimiento. Estos resultados concuerdan con los obtenidos por Espinosa-Calderón et al. (2012), quienes detectaron diferencias en la longitud de la mazorca y los granos por mazorca en el sentido de que los materiales evaluados presentaron una disminución en estas variables y por tanto su rendimiento en grano se vio afectado.

Para días a floración masculina, el grupo 2 tuvo mayor precocidad con respecto al grupo 1 y este último que el 
grupo 3 con 4 d de diferencia entre ellos, respectivamente. Los híbridos testigos tuvieron su floración a partir de los $80 \mathrm{~d}$. En altura de planta y mazorca, el grupo 3 tuvo una marcada diferencia mayor al tratarse de materiales más altos con respecto al grupo 2 y éste con respecto al grupo 1, de porte bajo. Este resultado es similar al obtenido por Tadeo-Robledo et al. (2016), quienes registraron una floración masculina de híbridos de 80 d, en contraste con 78 $\mathrm{d}$ de sus cruzas, lo que demuestra que las cruzas simples son más precoces.

El rendimiento de grano por hectárea de las mejores líneas evaluadas fue desde $3.405 \mathrm{t} \mathrm{ha}^{-1}$ hasta $4.479 \mathrm{t} \mathrm{ha}^{-1} \mathrm{y}$ buen comportamiento de todas las variables estudiadas para los tres ambientes de producción (Cuadro 5). Espinosa et al. (2003) mencionan que una línea de maíz para ser utilizable como progenitor femenino en híbridos de cruza simple debe producir rendimientos de un mínimo de $3.0 \mathrm{t} \mathrm{ha}^{-1}$, por lo que el rendimiento que expresaron las 10 mejores líneas evaluadas les confiere un gran potencial y podrían ser utilizadas como progenitores de híbridos de cruza simple en programas de mejoramiento genético, por que presentaron rendimientos de más de $3 \mathrm{t} \mathrm{ha}^{-1}$.

La línea L39 mostró el promedio más alto para las variables estudiadas, con rendimiento por hectárea de $4.4 \mathrm{t} \mathrm{ha}^{-1}$ y comportamiento similar en los tres ambientes. También sobresalieron, las líneas $L 43$ y $L 40$, con rendimientos por hectárea de $3.939 \mathrm{t} \mathrm{ha}^{-1}$ y $3.840 \mathrm{t} \mathrm{ha}^{-1}$, respectivamente, y un buen comportamiento en las variables evaluadas. Esto da evidencia del comportamiento estable de las líneas para usarse como progenitoras hembra, aspecto fundamental para el éxito de la producción, rentabilidad económica y técnica en las empresas semilleras (Vallejo et al., 2008).

Aunque los híbridos comerciales HS-2 y H-40 tuvieron promedios más altos que todas las cruzas evaluadas, tres cruzas simples $(99 \times 107,107 \times 206$ y $106 \times 180)$ fueron sobresalientes, ya que tuvieron rendimientos de alrededor de $8 \mathrm{t} \mathrm{ha}^{-1}$, una tonelada menos que los híbridos, pero fueron mucho más precoces que estos últimos, con un promedio de 73 días a floración en comparación con los 80 d que presentaron los híbridos comerciales trilineales.

Las cruzas simples de mayor rendimiento en general fueron más precoces que los dos híbridos comerciales (Cuadro 5); al respecto, Virgen-Vargas et al. (2014), señalan que las cruzas simples en general son más precoces que las líneas progenitoras. La precocidad de las cruzas simples se debe en parte a que en el proceso de mejoramiento para obtener las líneas se eliminaron las plantas más tardías y menos altas, las cuales no soportaron la competencia de las plantas precoces que crecieron más rápido.
Esto permitió que las plantas seleccionadas resultaran con igual precocidad y altura que la variedad original, pero con mayor capacidad de rendimiento, lo que concuerda con lo señalado por Morales et al. (2007) de que el rendimiento de grano y la precocidad son las variables más importantes para definir la estructura de la variabilidad fenotípica entre materiales genéticos adaptados y exóticos.

De las cruzas simples de mayor rendimiento se identificaron tres $(99 \times 107,107 \times 206$ y $106 \times 180)$ que podrían usarse como híbridos comerciales porque presentaron rendimientos similares a los híbridos testigo, pero fueron más precoces. Arellano et al. (2011) mencionan que ésto es debido a que la floración presenta una mayor uniformidad porque las cruzas simples están formadas con líneas con entre cinco y siete autofecundaciones.

\section{CONCLUSIONES}

El rendimiento de semilla de las 10 mejores líneas evaluadas fue superior a $3.4 \mathrm{t} \mathrm{ha}^{-1}$, por lo cual podrían ser utilizadas como líneas progenitoras de híbridos de cruza simple.

Las cruzas simples $(99 \times 107,107 \times 206$ y $106 \times 180)$ tienen potencial para usarse como híbridos comerciales porque sus rendimientos resultaron competitivos con los de los híbridos testigo y además sus líneas progenitoras mostraron buen rendimiento de semilla. En caso de utilizarse como cruzas simples, estos materiales tienen la ventaja con respecto a los híbridos HS-2 y H-40, de que requieren un ciclo agrícola menos para obtener y mantener su semilla, por lo que podrían reducir el costo de la semilla híbrida para el productor.

Las cruzas simples sobresalientes pueden también utilizarse para la formación de híbridos trilineales combinándolas con líneas sobresalientes.

\section{BIBLIOGRAFÍA}

Andrade F., A. Cirilo, S. Uhart y M. E. Otegui (1996) Ecofisiología del Cultivo de Maíz. Editorial La Barrosa y Dekalb Press. Buenos Aires, Argentina. 292 p.

Arellano V. J. L., J. Virgen V., I. Rojas M. y M. A. Ávila P. (2011) H-70: Híbrido de maíz de alto rendimiento para temporal y riego del Altiplano Central de México. Revista Mexicana de Ciencias Agrícolas 2:619-626

Ávila P. M. A., J. L. Arellano V., J. Virgen V. y A. J. Gámez V. (2009) H-52 Híbrido de maíz para Valles Altos de la Mesa Central de México. Agricultura Técnica en México 35:237-240.

Boomsma C. R., J. B. Santini, M. Tollenaar and T. J. Vyn (2009) Maize morphophysiological responses to intense crowding and low nitrogen availability: an analysis and review. Agronomy Journal 101:1426-1452.

Cervantes-Santana T., M. A. Oropeza-Rosas y D. Reyes-López (2002) Selección para rendimiento y heterosis de líneas endogámicas de maíz irradiado. Agrociencia 36:421-431. 
Cuadro 5. Comparación de medias de ocho variables de las mejores 10 y peores cinco líneas per se de maíz, seis cruzas simples y testigos evaluados en tres ambientes.

\begin{tabular}{|c|c|c|c|c|c|c|c|c|}
\hline Material & $R G$ & DFM & AP & AM & LMz & $\mathrm{DMz}$ & $\mathrm{NHM}$ & $\mathrm{NGH}$ \\
\hline & \multicolumn{8}{|c|}{ Mejores líneas } \\
\hline L39 & $4479.50 \mathrm{a}$ & $75.30 q-u$ & $213.30 \mathrm{a}$ & $112.30 \mathrm{~b}$ & $15.30 \mathrm{a}$ & $4.90 \mathrm{a}$ & 13.40 dce & $26.30 \mathrm{a}$ \\
\hline$\llcorner 43$ & $3939.50 \mathrm{~b}$ & $73 x-c$ & 180.40 k-n & $73.80 \mathrm{vxw}$ & $13.50 j$ & $4.60 \mathrm{cb}$ & 13.40 dce & $24.60 \mathrm{cb}$ \\
\hline$\llcorner 40$ & $3840.20 \mathrm{c}$ & $76.80 \mathrm{~g}-\mathrm{O}$ & $215 a$ & $118.10 \mathrm{a}$ & 13.010 & 4.50 cde & $12.40 \mathrm{j}-\mathrm{m}$ & $23.80 \mathrm{de}$ \\
\hline$\llcorner 41$ & $3795.50 \mathrm{dc}$ & $77.70 c-j$ & $211.20 \mathrm{ba}$ & $101.90 \mathrm{c}-\mathrm{f}$ & $14.30 \mathrm{~d}$ & $4.70 \mathrm{~b}$ & 13.20 dfe & $26.90 \mathrm{a}$ \\
\hline L29 & 3739.30 de & $73.70 w-b$ & 178.70 nm & $85.80 \mathrm{sqr}$ & $14.50 \mathrm{c}$ & $4.20 \mathrm{f}-\mathrm{j}$ & $10.90 \mathrm{u}$ & $22.90 \mathrm{fgh}$ \\
\hline L52 & 3708.70 de & 74.20 t-y & $199.80 \mathrm{c}$ & 88.90 n-q & $12.50 \mathrm{u}$ & $4.60 \mathrm{cb}$ & $12.70 \mathrm{~g}-\mathrm{i}$ & $22.10 \mathrm{i}-\mathrm{I}$ \\
\hline L9 & $3679.60 \mathrm{e}$ & $75.90 \mathrm{l}-\mathrm{r}$ & $195.10 \mathrm{de}$ & $101.80 \mathrm{c}-\mathrm{f}$ & $13.70 \mathrm{fg}$ & $4.40 \mathrm{de}$ & $12.60 \mathrm{~h}-\mathrm{k}$ & $23.00 \mathrm{fg}$ \\
\hline$\llcorner 47$ & $3576.80 \mathrm{f}$ & $73.10 w-c$ & 193.40 de & $85.90 \mathrm{sqr}$ & $14.20 \mathrm{~d}$ & $4.60 \mathrm{~cd}$ & $13.50 \mathrm{dc}$ & 23.40 fde \\
\hline L31 & $3495.50 \mathrm{gf}$ & 77.80 b-i & 168.80 pqr & $88.90 n-q$ & $13.60 \mathrm{ghi}$ & $4.20 \mathrm{f}-\mathrm{j}$ & $11.30 \mathrm{st}$ & $23.90 \mathrm{de}$ \\
\hline \multirow[t]{2}{*}{$\llcorner 44$} & $3405.50 \mathrm{~g}$ & $72.70 y-c$ & $185.70 \mathrm{~g}-\mathrm{k}$ & $99.80 \mathrm{e}-\mathrm{i}$ & $13.40 \mathrm{k}$ & 4.40 de & $12.90 \mathrm{gfh}$ & 20.80 qop \\
\hline & \multicolumn{8}{|c|}{ Peores líneas } \\
\hline L38 & $1138.10 \mathrm{bc}$ & $78.30 \mathrm{~b}-\mathrm{g}$ & 180.40 k-n & $86 \mathrm{sqr}$ & $11.80 \mathrm{w}$ & $3.50 t$ & $11.30 \mathrm{st}$ & $19.70 \mathrm{~s}$ \\
\hline L30 & $1104.50 \mathrm{c}$ & 76.40 h-p & 153.50 uv & $70.30 y x$ & $10.20 \mathrm{c}$ & $3.70 \mathrm{qr}$ & 12.10 no & $14.90 \times w$ \\
\hline L12 & $945.80 \mathrm{~d}$ & $75.70 \mathrm{~m}-\mathrm{t}$ & 183.80 h-m & 78.10 vuw & $11.00 \mathrm{a}$ & $3.60 \mathrm{srt}$ & $11.30 \mathrm{st}$ & $14.50 x$ \\
\hline L15 & $614.90 \mathrm{e}$ & $77.30 \mathrm{e}-\mathrm{I}$ & $142.60 w x$ & $73.40 \times w$ & $11.10 z$ & $3.60 \mathrm{st}$ & 11.20 poq & $16.04 \mathrm{vu}$ \\
\hline L3 & $540.60 \mathrm{e}$ & $77.40 \mathrm{~d}-\mathrm{I}$ & $187 \mathrm{f}-\mathrm{j}$ & $84.90 \mathrm{sqr}$ & $11.20 y$ & $3.60 \mathrm{sr}$ & $11.60 \mathrm{srq}$ & $11.20 y$ \\
\hline \multirow[t]{2}{*}{$\mathrm{DSH}_{0.05}$} & 95.50 & 1.60 & 5.90 & 5.09 & 0.11 & 0.10 & 0.36 & 0.70 \\
\hline & \multicolumn{8}{|c|}{ Cruzas } \\
\hline $99 \times 107$ & $8432.40 \mathrm{c}$ & $72.40 \mathrm{~cd}$ & $219.30 b$ & $108.90 \mathrm{~cd}$ & $15.70 \mathrm{~d}$ & $4.70 \mathrm{~d}$ & $12.40 \mathrm{~d}$ & $27.60 \mathrm{~d}$ \\
\hline $107 \times 206$ & 8419.5 c & $73 \mathrm{cbd}$ & $226 a$ & $115.40 \mathrm{~b}$ & $16.40 \mathrm{a}$ & $4.60 \mathrm{e}$ & $12.40 \mathrm{~d}$ & $27.40 d$ \\
\hline $106 \times 180$ & $8276.50 \mathrm{~d}$ & $74 b$ & $212.40 \mathrm{c}$ & $105.30 \mathrm{~d}$ & $15.20 \mathrm{f}$ & $4.60 \mathrm{ed}$ & $12.80 \mathrm{c}$ & $25.00 \mathrm{e}$ \\
\hline $105 \times 194$ & 7606.60 e & $71.90 \mathrm{~d}$ & $223.70 \mathrm{ba}$ & $113.10 \mathrm{cb}$ & $15.60 \mathrm{e}$ & $4.70 \mathrm{~d}$ & $12.70 \mathrm{dc}$ & $24.70 \mathrm{e}$ \\
\hline $109 \times 205$ & $7241.60 \mathrm{f}$ & $73.10 \mathrm{cb}$ & $223.80 \mathrm{ba}$ & $134.10 \mathrm{a}$ & $15.90 \mathrm{c}$ & $4.70 \mathrm{~d}$ & $12.70 \mathrm{dc}$ & $27.40 \mathrm{~d}$ \\
\hline \multirow[t]{2}{*}{$142 \times 188$} & $6515.20 \mathrm{~g}$ & $73.30 \mathrm{cb}$ & $196.10 d$ & $100 \mathrm{e}$ & $14.90 \mathrm{~g}$ & $4.80 \mathrm{c}$ & $13.30 \mathrm{~b}$ & $28.10 \mathrm{c}$ \\
\hline & \multicolumn{8}{|c|}{ Testigos } \\
\hline HS-2 & $9421.50 \mathrm{a}$ & $80.80 \mathrm{a}$ & 227.80 a & $116.90 \mathrm{~b}$ & $16.00 \mathrm{c}$ & $5.20 \mathrm{~b}$ & $17.02 \mathrm{a}$ & $34.00 \mathrm{a}$ \\
\hline $\mathrm{H}-40$ & 8913.70 b & $80.50 \mathrm{a}$ & $218.80 \mathrm{~b}$ & $107.10 \mathrm{~d}$ & $16.20 \mathrm{~b}$ & $5.40 \mathrm{a}$ & $17.00 \mathrm{a}$ & $30.80 \mathrm{~b}$ \\
\hline $\mathrm{DSH}_{0.05}$ & 145.40 & 1.12 & 5.15 & 5.04 & 0.09 & 0.09 & 0.40 & 0.50 \\
\hline
\end{tabular}

En columnas, letras iguales, indican no diferencias dentro del grupo de genotipos (Tukey, 0.05). RG: rendimiento de grano por hectárea (t ha-1 ${ }^{-1}$; DFM: días a floración masculina; AP. altura de planta (cm); AM: Altura de mazorca (cm); LMz: longitud de mazorca (cm); DMz: diámetro de mazorca (cm); NHM: número de hileras por mazorca; NGH: número de granos por hilera; DSH: diferencia significativa honesta (0.05). 
De Brito C. H., D. Lima S., A. María B., L. Savelli G. y M. T. Gomes L. (2011) Reducción del área foliar en maíz en una región tropical en Brasil y sus efectos en caracteres agronómicos. Interciencia 36:291295.

Espinosa C. A., M. Sierra M. y N. Gómez M. (2003) Producción y tecnología de semillas mejoradas de maíz por el INIFAP en el escenario sin la PRONASE. Agronomía Mesoamericana 14:117-121.

Espinosa-Calderón A., M. Tadeo-Robledo, I. Arteaga-Escamilla, A. Turrent-Fernández, M. Sierra-Macías, N. Gómez-Montiel, A. Palafox-Caballero, R. Valdivia-Bernal, V. Trejo-Pastor y E. Canales-Islas (2012) Rendimiento de las generaciones $F_{1}$ y $F_{2}$ de híbridos trilineales de maíz en los Valles Altos de México. Universidad y Ciencia 28:57-64.

Fan X. M., J. Tan, H. M. Chen and J. Y. Yang (2003) Heterotic grouping for tropical and temperate maize inbreds by analyzing combining ability and SSR markers. Maydica 48:251-257.

FAOSTAT, Organización de las Naciones Unidas para la Alimentación y la Agricultura (2015) Base de datos Estadísticos. Roma. http://www. fao.org/faostat/es/\#data/QC. (Abril 2017)

Gómez-Espejo A. L., J. D. Molina-Galán, J. J. García-Zavala, M. C. MendozaCastillo y A. de-la-Rosa-Loera (2015) Poblaciones exóticas originales y adaptadas de maíz. I: variedades locales de clima templado x variedades tropicales. Revista Fitotecnia Mexicana 38:57-66.

Goodman M. M. (1992) Choosing and using tropical corn germplasm. Annual Corn and Sorghum Research Conference Proceedings. 47:47-64.

Hallauer A. R. (1992) Registration of BS27 maize germplasm. Crop Science 32:1512-1513.

Hallauer A. R. (1994) Registration of BS28 and BS29 maize germplasm. Crop Science 34:544-545.

Hallauer A. R., W. A. Russell and K. R. Lamkey (1988) Corn breeding. In: Corn and Corn Improvement. G. F. Sprague and J. W. Dudley (eds.). American Society of Agronomy, Crop Science Society of America, Soil Science Society of America, Madison. WI. USA. pp:463-564.

Hallauer A. R., M. J. Carena and J. B. Miranda F. (2010) Quantitative Genet- ics in Maize Breeding. Springer Science+Business Media. New York, NY, USA. $664 \mathrm{p}$.

Luna M. B. M., M. A. Hinojosa R., O. J. Ayala G., F. Castillo G. y J. A. Mejía C. (2012) Perspectivas de desarrollo de la industria semillera de maíz en México. Revista Fitotecnia Mexicana 35:1-7.

Morales R. M. M., J. Ron P., J. J. Sánchez G., J. L. Ramírez D., L. Cruz L., S. Mena M., S. Hurtado P. y M. Chuela B. (2007) Relaciones fenotípicas y heterosis entre híbridos comerciales y germoplasma exótico de maíz en Jalisco, México. Revista Fitotecnia Mexicana 30:285-294.

Pérez-López F. J., R. Lobato-Ortiz, J. J. García-Zavala, J. D. Molina-Galán, J. J. López-Reynoso y T. Cervantes-Santana (2014) Líneas homocigóticas de maíz de alto rendimiento como progenitoras de híbridos de cruza simple. Agrociencia 48: 425-437.

Ramírez D. J. L., J. J. Wong P., J. A. Ruiz C. y M. Chuela B. (2010) Cambio de fecha de siembra del maíz en Culiacán, Sinaloa, México. Revista Fitotecnia Mexicana 33:61-68.

SAS Institute (2002) User's Guide of SAS. SAS Insitute Inc. Cary, N. C., USA. $550 \mathrm{p}$

Tadeo-Robledo M., J. Zaragoza-Esparza, A. Espinosa-Calderón, A. TurrentFernández, B. Zamudio-Gónzalez, J. Virgen-Vargas, K. Y. Mora-García y R. Valdivia-Bernal (2016) Productividad de la generación $F_{7}$ y $\mathrm{F}_{2}$ de híbridos de maíz (Zea mays $L$.) de valles altos de México. Agrociencia 50:33-41.

Vallejo D. H. L., J. L. Ramírez D., M. Chuela B. y R. Ramírez Z. (2008) Manual de producción de semilla de maíz. Estudio de caso. Folleto Técnico Núm. 14. Campo Experimental Uruapan. INIFAP, CIRPAC. Guadalajara, Jalisco, México. 96 p.

Virgen-Vargas J., R. Zepeda-Bautista, M. A. Ávila-Perches, A. Espinosa-Calderón, J. L. Arellano-Vázquez y A. J. Gámez-Vázquez (2014) Producción de semilla de líneas progenitoras de maíz: densidad de población e interacción. Agronomía Mesoamericana 25:323-335.

Virgen-Vargas J., R. Zepeda-Bautista, M. A. Ávila-Perches, I. Rojas-Martínez, A. Espinosa-Calderón y A. J. Gámez-Vázquez (2016) Desespigamiento en cruzas simples progenitoras de híbridos de maíz (Zea mays L.) para Valles Altos de México. Agrociencia 50:43-59. 